Álvarez-Núñez, Q., López-Gómez, S., Parada-Gañete, A. \& Gonçalvez, D. (2021). Cultura profesional y TIC en la formación del profesorado en tiempos de crisis: la percepción de los docentes. Revista Electrónica Interuniversitaria de Formación del Profesorado, 24(2), 153- 165.

\title{
Cultura profesional y TIC en la formación del profesorado en tiempos de crisis: la percepción de los docentes
}

\author{
Quintín Álvarez Núñez ${ }^{(1)}$, Silvia López Gómez ${ }^{(1)}$, Ana Parada Gañete ${ }^{(1)}$, Daniela Gonçalves ${ }^{(2)}$ \\ (1) Universidade de Santiago de Compostela (USC), ${ }^{(2)}$ ESE de Paula Frassinetti, CEDH da UCP; \\ CIPAF da ESEPF (Portugal)
}

\section{Resumen}

En este artículo analizamos algunas razones básicas que explicarían las dificultades que el profesorado y los centros de Primaria han tenido para adaptarse a los cambios producidos por la pandemia. Para ello, nos basaremos en los resultados de dos investigaciones del grupo Stellae (USC). De la primera, utilizaremos los datos de las entrevistas a docentes de las Facultades de la USC que imparten la titulación de Magisterio, recogiendo sus percepciones sobre la construcción del conocimiento y la cultura profesional. De la segunda, usaremos los resultados de las entrevistas a un grupo de profesores especialistas en TIC, junto con las entrevistas realizadas a docentes de dos centros seleccionados por sus buenas prácticas, analizando sus percepciones sobre el uso escolar de las TIC y los Materiales Didácticos Digitales. Terminaremos con un breve análisis de cómo han de cambiar los procesos de formación docente, para hacer frente a las incertidumbres y nuevos desafíos que afronta la escuela en tiempos de crisis.

\section{Palabras clave}

Cultura profesional docente; TIC; Materiales Didácticos Digitales; Pandemia COVID 19.

\section{Contacto:}

Silvia López Gómez, silvialopez.gomez@usc.es, Av. Ramón Ferreiro, s/n, 27002, Lugo.

El artículo toma datos de dos proyectos de investigación: “Desarrollo del conocimiento profesional a través del plan de estudios del grado de maestro en educación primaria. Perspectivas del alumnado y profesorado" (EDU2012-39866-C02-01, Ministerio de Economía y Competitividad) y "La escuela de la sociedad digital: análisis y propuestas para la producción y uso de los contenidos digitales educativos" (EDU2015-64593-R, Ministerio de Economía y Competitividad). 


\title{
Professional culture and ICT in teacher education in times of crisis: the perception of teachers
}

\begin{abstract}
In this paper, we analyzed some reasons that would explain the difficulties that teachers and primary schools had in adapting to the changes produced by the COVID 19 pandemic. For this, we start from the results of two investigations by the Stellae group (USC). From the first, we will use the data from the interviews with professors from the Faculties of the USC that confer the degree of Teaching, collecting their perceptions about the construction of knowledge and professional culture. From the second, we will use the results of interviews with a group of ICT teachers, together with interviews and observations made with teachers from two centers selected for their good practices, analyzing their perceptions about the school use of ICT and Materials Digital didactics. We will end with a brief analysis of how teacher training processes must change, in order to deal with the uncertainties and new challenges that the school faces in times of crisis.
\end{abstract}

\section{Key words}

Professional teaching culture; ICT; Digital Teaching Materials; COVID 19 pandemic.

\section{Introducción}

La pandemia de la Covid-19, durante el período de confinamiento, ha cambiado, al menos temporalmente, nuestro modelo de enseñanza, sustituyendo un proceso hasta ese momento esencialmente presencial por otro inevitablemente on-line. Lo cual ha traído, durante este período, importantes consecuencias: ha cambiado el papel de las TIC haciendo que éstas pasasen de ser, en muchos casos, un simple apoyo a la docencia a convertirse en una parte esencial e ineludible del proceso de enseñanza-aprendizaje, configurándose como un recursos indispensable durante el período de confinamiento para mantener el contacto diario con todo el alumnado, suponiendo nuevas formas de aprender y enseñar, tanto para el alumnado como para el profesorado (Ferreira \& Gonçalves, 2020); ha revelado la enorme brecha digital existente en los diversos territorios y áreas y entre las familias de altas y bajas rentas, así como las carencias dotacionales de muchos centros; ha obligado al profesorado en general, y de Educación Primaria en particular, a enfrentarse con un recurso que no dominaban; ha convertido a las familias en colaboradoras necesarias para el proceso de enseñanza-aprendizaje de sus hijo/as, etc.

Siguiendo los estudios de García-Fernández et al. (2020), Equipo de traballo de Nova Escola Galega (2020) y Bermello (2020), entre otros, podemos señalar las dificultades más relevantes que el profesorado y los centros de Primaria han tenido para adaptarse a los cambios producidos por la pandemia: la existencia de una profunda brecha digital entre el alumnado en función de su contexto social (rural o urbano) y las posibilidades económicas de sus familias, ésta se refiere tanto a la carencia u obsolescencia de los ordenadores como a la no disposición o dificultad de acceso a una red de calidad, al escaso o nulo dominio técnico de algunos padres con dificultades para manejar los ordenadores y/o acceder a las plataformas propuestas por los centros para la actividad formativa y la inexistencia, en algunas casas, de otro tipo de hardware, como impresoras y escáneres, necesarios para la realización de las actividades 
propuestas y su devolución a los docentes; la ampliación de los horarios de atención por parte del profesorado, que, debido a estas limitaciones, en ocasiones tenían que alargarse excesivamente, para dar respuesta a las dificultades de muchos estudiantes; el importante papel jugado por la familia en este contexto, con su atención y sus estrategias de acompañamiento de sus hijos/as y la impotencia, preocupación y angustia que sintieron algunos padres y madres, con bajo nivel escolar, al sentirse incapaces de acompañar y dar soporte a sus hijos en la realización de sus tareas escolares; la necesidad de las familias y los alumnos de elaborar nuevas rutinas y hábitos de trabajo, en un entorno, como el hogar, cuando éstas están habitualmente articuladas en las aulas y los centros; la tensión y el estrés de las familias para combinar el trabajo on-line con la atención a sus hijos en un contexto de fuerte incertidumbre y preocupación por su futuro laboral; el riesgo, en los centros donde no existió una buena coordinación entre el profesorado y cada docente mandó sus propias actividades, de una sobrecarga de tareas telemáticas, que sólo generan frustración y ansiedad en los niños/as y las familias; las consecuencia del cierre de los centros y de la necesidad de trabajar on-line afectó, de un modo particular, al que tiene necesidades educativas de atención específica, que pueden carecer de la competencia digital para trabajar on-line de forma autónoma y necesitan el contacto directo, la presencia, el apoyo y el estímulo del profesorado, a lo cual se unió la insuficiencia e inadecuación del material digital adaptado para este tipo de alumnado; las dificultades de alguna plataforma educativas para hacer frente a un gran número de participaciones en un período de tiempo limitado, al no estar pensadas para satisfacer una demanda tan amplia; la falta de formación y experiencia previa de muchos docentes para poder impartir con eficacia, la docencia telemática; la carencia, en general, de directrices y orientaciones claras por parte de la Administración educativa y de apoyos útiles por parte de ella y sus servicios; la necesidad de crear materiales específicos para explicar la problemática del COVID al alumnado, que dio lugar a una gran cantidad y variedad de materiales didácticos, de diversos tipos y en distintos formatos, (Rodríguez-Rodríguez, et al. 2020); etc.

Ante todos estos cambios temporales, parece evidente que los centros y el profesorado no estaban preparados para asumirlos.

Este artículo pretende dar una primera respuesta a las razones de esta situación, más allá del hecho obvio de que es difícil estar preparado para un acontecimiento tan excepcional. Para ello utilizamos dos conceptos claves: las TIC y su papel en los centros educativos y la cultura profesional, centrada en la formación del profesorado. Los analizaremos utilizando los datos procedentes de dos investigaciones conectadas con ambos conceptos. Así exploramos algunas de las razones por las cuales los profesores de primaria, en general, por su formación, su cultura y su experiencia profesional no estaban bien preparados para asumir, con eficacia, el reto que supuso la docencia on-line durante los meses que duró el confinamiento.

Comenzaremos, entonces, por definir el concepto de cultura profesional educativa, el cual está directamente asociado a la forma en que el profesorado desarrolla su trabajo en y para la escuela (Víton de António \& Gonçalves, 2017). Nos referimos a un modo de relación que se traduce en las prácticas que utilizan, las creencias que subyacen en su forma de entender el trabajo que realizan, así como las rutinas que construyen en su relación con la escuela, con otros docentes y con los alumnos. Esta cultura profesional refleja en parte el proceso de socialización del yo en la profesión (Dubar, 1997), así como su sentido de pertenencia a la misma y a la propia escuela. Teniendo en cuenta que la identidad se construye a partir del proceso de socialización en una organización, aquí en la institución escolar, consideramos que el ingreso a la carrera docente, así como el desarrollo profesional de los profesores, está íntimamente ligado a la cultura profesional predominantes tanto en la escuela como en el grupo disciplinar al que pertenecen (Marcelo, 2009). Así, dependiendo del estilo de cultura 
imperante en la organización escolar, las reglas, las creencias, la forma de actuar serán asimiladas o no por los docentes y tendrán claras repercusiones a nivel de su "ser docente".

Por otra parte, la integración educativa de las TIC ha avanzado de manera considerable en los últimos años, pero sigue persistiendo la necesidad de seguir avanzando en la articulación de políticas educativas destinadas a facilitar la innovación didáctica con las tecnologías digitales (Area et al., 2020) ya que, como evidencian numerosos trabajos e informes internacionales (por ejemplo: European Schoolnet, 2006; Ranguelov et al., 2011; Kopcha, 2012), la introducción de las tecnologías en las aulas no siempre estuvo acompañada por los necesarios cambios metodológicos (Gros et al., 2020).

Lo que está claro es que la combinación de ambos retos: la integración de las TIC en las aulas y la necesaria innovación didáctica que ello requiere, suponen una transformación digital de las organizaciones educativas, entendido este hecho como un proceso complejo, disruptivo y en el que se entrecruzan factores de distinta naturaleza (tecnológicos, organizativos y culturales) (Matt et al., 2015; Leignel et al., 2016; Gale \& Aarons, 2018). En esta dirección, Area et al. (2020) recogen la existencia de diferentes estudios que ponen en evidencia las dificultades existentes en los centros educativos para iniciar, desarrollar y consolidar una auténtica transformación digital. Como veremos posteriormente, muchas de ellas aparecen también en las investigaciones que aquí recogemos. Hemos agrupado las conclusiones de tres de ellos (Balanskat et al., 2006; British Educational Communications and Technology Agency, 2004; Hew \& Brush, 2007) en tres dimensiones de análisis clave en relación a:

(a) El profesorado: ausencia de competencia digital, carencia de dominio para el uso pedagógico de la tecnología, baja motivación, falta de confianza, carencia de habilidades de liderazgo, formación inadecuada del profesorado y actitudes y creencias negativas ante la tecnología.

(b) Las infraestructuras TIC: baja calidad y mantenimiento inadecuado del hardware, acceso limitado a equipos de TIC, falta de capacitación en el uso del software.

(c) La cultura escolar: currículum disciplinar, estructuras organizativas rígidas, metodologías de enseñanza y evaluaciones tradicionales, resistencia al cambio, falta de un plan de integración e innovación tecnológica y ausencia de experiencias en el desarrollo de proyectos educativos apoyados en el uso de las TIC.

En general, esta preocupación por el estudio y análisis del uso de las tecnologías en las aulas tiene una presencia notoria en las investigaciones sobre educación. Véase a este respecto, por ejemplo, los trabajos de Gonçalves et al. (2015); Gómez-Mendoza et al. (2016); RodríguezRodríguez \& Rodríguez-Regueira (2016); Peirats et al. (2018). De estos estudios se desprende que la utilización de recursos digitales requiere de destrezas metodológicas y tecnológicas que no todo el profesorado tiene, inciden en la necesidad de una capacitación pedagógica y de una formación que posibilite el saber diseñar, seleccionar o adaptar los MDD a diferentes realidades o necesidades que puedan surgir en las aulas.

\section{Método}

El primero de los proyectos de investigación en los que se basa este artículo es el titulado "Desarrollo del conocimiento profesional a través del plan de estudios del grado de maestro en educación primaria. Perspectivas del alumnado y profesorado" (CP). Su finalidad era 
identificar, analizar e interpretar las percepciones de los futuros profesores y sus formadores sobre los conocimientos que están construyendo en el desarrollo del plan de estudios de maestro en educación primaria y la contribución de éstos a configurar una identidad profesional. En él participaron los grupos de investigación Stellae (USC) y FODIP (UB). Se desarrolló desde el año 2012 hasta el 2016.

Siguiendo la propuesta de Rodríguez, Gil y García (1999), el proceso de investigación se desarrolló en 4 fases:

(1) Fase preliminar: selección y análisis de fuentes documentales para conocer el marco legal, diseño curricular y marco institucional de los planes de estudio del Grado de Primaria en la USC y recoger la información básica sobre profesorado y alumnado del grado y tutores del prácticum.

(2) Fase preparatoria, en la que se realizó: a) selección de los participantes en el estudio, utilizando, de forma combinada, un muestreo intencional y de casos típicos, para contar con la participación de sujetos representativos; b) elaboración del diseño del guion de los relatos, las entrevistas y los grupos de discusión.

(3) Recogida de datos, a través de tres técnicas: los relatos, la entrevista en profundidad y los grupos de discusión. Es en la entrevista al profesorado seleccionado en el que basamos los análisis realizados en este artículo. En concreto, fueron 13 profesores, 6 de la facultad de Santiago y 7 de la de Lugo.

(4) Fase analítica: analizando cualitativamente los datos recogidos, a través del programa Atlas-ti (versión 7).

A su vez, en el proyecto "La escuela de la sociedad digital: análisis y propuestas para la producción y uso de los contenidos digitales educativos" (ED) se analizó el estado de los MDD dirigidos a la Educación Primaria en una muestra de tres comunidades autónomas (Canarias, Galicia y Valencia). Fue desarrollado por tres grupos universitarios de investigación (EDULLAB, Stellae y CRIE) desde el año 2016 hasta finales del 2019. Los estudios que conformaron el proyecto fueron:

(1) Estudio I. Analizar las características técnicas y pedagógicas de una muestra de plataformas y repositorios de MDD.

(2) Estudio II. Identificar las opiniones de distintos agentes educativos sobre el tránsito de los libros de texto en papel a los contenidos o materiales digitales.

(3) Estudio III. Examinar el uso de los MDD en las aulas, explorando su impacto en los procesos de enseñanza y aprendizaje en una muestra de centros educativos de las comunidades de Canarias, Galicia y Valencia.

(4) Estudio IV. Elaborar una guía para la producción y uso de MDD, con recomendaciones para el profesorado, las familias y empresas productoras.

Este artículo se centra en parte de los resultados de los estudios II y III. Pretendemos analizar las valoraciones que el profesorado gallego de primaria tiene sobre el potencial educativo de las TIC y los MDD y recoger el uso que de ellos realizan en sus aulas. 
La muestra la conforma profesorado de $5^{\circ}$ y $6^{\circ}$ de Primaria de dos centros públicos de Galicia, seleccionados por sus buenas prácticas con tecnologías.

Para la recogida de información se empleó la entrevista semiestructurada en profundidad.

En este trabajo se analiza una entrevista grupal en la que participaron 5 profesores, de distintos centros educativos, públicos y privados, y 9 entrevistas individuales realizadas a docentes, equipo directivo y coordinadores TIC, pertenecientes a los dos centros seleccionados.

Para el análisis de la información de estas entrevistas, comenzamos estableciendo las principales dimensiones de análisis: el papel de la formación en la construcción de la cultura profesional y la valoración, las fortalezas y debilidades que el profesorado señala en relación con las TIC. Posteriormente, se realizó una selección de los fragmentos de las entrevistas en las que son tratados estas dimensiones, para obtener las visiones del profesorado en cada proyecto. La foto obtenida se muestra en el siguiente apartado.

\section{Resultados}

A la hora de valorar la formación que reciben los/as futuros/as maestros/as, partimos de que ésta es una variable fundamental para la construcción y desarrollo de su cultura profesional, y por consiguiente, para la comprensión y mejora de su práctica docente. Ahora bien, en esa formación inicial hemos de señalar la existencia de limitaciones organizativas, docentes $e$ institucionales que condicionan el desarrollo y la calidad de la formación que se imparte. En el proyecto sobre conocimiento profesional, el profesorado universitario participante destaca algunas que vamos a exponer seguidamente.

En cuanto a las organizativas, se detecta una evidente falta de coordinación docente y una fuerte tendencia al trabajo individual y aislado de cada profesor/a en su aula y con su alumnado: "La coordinación es difícil, los departamentos atienden más a la burocracia que a ella y a crear el clima que la pueda facilitar [...] De tal modo que las facultades de educación son como una central eléctrica, con una energía que se desperdicia tontamente porque no se trabaja interrelacionadamente" (CP, entrevista Pr. A, p. 13 y 15). Este trabajo aislado y esta falta de colaboración entre el profesorado de la Facultad de Educación se proyectará también en las escuelas, donde la coordinación se produce solo de un modo muy escaso y puntual, cuando existen profesores que tienen una cierta afinidad o bien cuando se aborda algún proyecto interdisciplinar. Pero, en el funcionamiento habitual de los centos, no suele existir esa interdisciplinariedad entre distintos docentes y grupos, así que esta coordinación es más algo excepcional y ocasional que general (ED, entrevista profesorado, p. 27). En cambio, los centros innovadores y con buenas prácticas tienden a caracterizarse por un mayor nivel de coordinación y colaboración entre su profesorado, como señala un docente de los dos centros seleccionados: "Tenemos claro que delegar es fundamental porque el trabajo en equipo es así, cada uno se encarga de una cosa y asume su responsabilidad" (ED, Entrevista Pr. B, p. 20).

Así mismo, se detecta que el modelo formativo está configurado por asignaturas compartimentalizadas y se configura como una enseñanza muy centrada en los contenidos escolares. Se transmiten contenidos que pueden estar actualizados, pero no se hace de manera que favorezca que el alumnado se apropie de ellos y se crea una distancia con lo que sucede en las escuelas, convirtiendo a éstas y la facultad en dos mundos muy separados.

En relación a las limitaciones docentes, el profesorado muestra resistencias al cambio que dificultan la implementación de innovaciones metodológicas y tecnológicas: "Aunque 
cambien los planes de estudio, es difícil cambiar la mentalidad del profesorado [...] Yo no voy a cambiar mi forma de pensar [...] Y, para cambiar la manera de actuar, hay que cambiar la manera de pensar" (CP, entrevista Pr. A, p. 22). Existe, en general, una metodología obsoleta. Ésta tiene que ver con el predominio de la teoría sobre la práctica, con la falta de experiencias concretas en las aulas y de una conexión más viva y directa con lo que se hace en las escuelas más allá de lo que el alumnado desarrolla en su estancia en los centros durante el prácticum. Se indica que hay que ir más a lo práctico, a que el alumnado aprenda haciendo.

Otra dificultad que limita las posibilidades de innovación y de mejora se debe a la existencia de una excesiva cantidad de alumnado en la facultad y a la escasez de profesorado y de recursos para satisfacer adecuadamente las necesidades docentes. Ello provoca que los grupos-clase sean de gran tamaño, con aulas masificadas, sobre todo en las clases expositivas, promoviendo un trabajo más formal y rutinario, basado en modelos didácticos más tradicionales y favoreciendo que en éstas se tienda a desarrollar un modelo de transmisión tradicional y magistrocéntrico de los contenidos (CP, Entrevista Pr. F., p. 11-12). Esto hace que los hábitos, la metodología y la cultura docente cambien con lentitud, y que las posibilidades de mejora se ralenticen. Existe el riesgo de que el alumnado, al no trabajar con metodologías innovadoras durante su formación inicial, se conviertan en profesionales con tendencia a reproducir el esquema tradicional que han vivido. Contribuyendo, así, a perpetuar estos modelos didácticos cuando vayan, como profesionales, a los centros educativos, ofreciendo una dificultad más para la innovación y el cambio. Todo ello estaría conectado, en primer lugar, con la dificultad para lograr una implementación adecuada de las TIC en la docencia y, en segundo, aunque los maestros las usen, tenderán a hacerlo de un modo más convencional, dificultando un uso más innovador y transformador de éstas en el proceso de enseñanzaaprendizaje.

En cuanto a las limitaciones institucionales, los docentes entrevistados destacan que los espacios disponibles son limitados y resultan insuficientes ante la gran cantidad de grupos de estudiantes. Los edificios son viejos, deteriorados, con pobreza visual y presentan carencias graves en cuanto a las necesidades docentes. Tampoco cuentan con el suficiente cuidado estético. A este respecto, nos dicen que: "Los alumnos serán creadores de espacios en el futuro y van a repetir la misma falta de cuidado y de estética que tiene el edificio de la facultad que "es viejo" y "se cae a pedazos" y, cuando se interviene en él, es para hacer "apaños" (CP, entrevista Pr. A, p. 14).

También nos indican, como una limitación importante, la existencia de horarios muy rígidos y poco flexibles, difíciles de modificar, que obstaculizan la introducción de dinámicas innovadoras y ayuda a que los profesores continúen trabajando con metodologías convencionales.

Por otra parte, parece claro, y así lo señalan diversos formadores, que el grado aporta una formación inicial que ya no es suficiente y hace que los futuros profesionales tengan que seguir formándose durante toda su vida profesional. Curiosamente, al entrevistar al profesorado de Educación Primaria, esta necesidad de formación permanente la perciben como un aspecto negativo, específicamente al hablar de la integración de las TIC en las aulas, dada la continua necesidad de actualización para conocer nuevos software, programas y aplicaciones, que cambian con frecuencia (ED, Entrevista grupal, p. 15 y 21).

En esta línea, los resultados obtenidos en el proyecto ED nos permiten conocer las percepciones de los docentes de Primaria en relación a la incorporación de las tecnologías en las aulas. A continuación, nos centramos en describir esas percepciones clasificándolas en fortalezas y debilidades para, finalmente, examinar la valoración que hacen de éstas a nivel general. 
Así, al hablar de fortalezas de las TIC, hacen referencia a una serie de aspectos que hemos agrupado en cuatro dimensiones y que describimos a continuación.

En primer lugar, el aumento de la motivación del alumnado debido, fundamentalmente, a lo atractivo que resultan para los/as niños/as el uso de recursos educativos digitales (RED), más adaptados a sus intereses y necesidades y por la sensación de que, en general, le son más próximos, los conocen y lo dominan.

En segundo lugar, la facilidad de acceso a los recursos y a la información y, a este respecto, señalan en varias ocasiones la inmediatez que supone el uso de los RED, incrementando exponencialmente la cantidad y calidad de éstos a disposición de los docentes.

En tercer lugar, destacan las funcionalidades de las TIC como un potente canal de comunicación y coordinación a varios niveles: alumno/a-alumno/a, docente-docente, docentealumno/a, docente-familia.

Por último, coinciden en situar como potencialidad la flexibilidad y capacidad de adaptación de los MDD. En este sentido, nos dicen:

Nos permiten a nosotros poder tomar decisiones que con los materiales tradicionales no podíamos, un libro de texto impreso está impreso y ahí no se puede tocar nada [...] El material digital ahora mismo nos está permitiendo adaptar posibilidades, incluso utilizar diferentes recursos para diferente tipo de alumnado dentro de la propia aula [...] (ED, Entrevista Pr. B, p. 2)

Por otro lado, las debilidades de las TIC indicadas por los docentes, las podemos agrupar en seis dimensiones:

La primera dificultad es la baja competencia digital de muchos docentes, que se manifiesta en las escasas habilidades para el uso de las TIC como recursos educativos en el aula y tiende a generar resistencias y rechazos hacia sus usos didácticos.

También señalan la velocidad y la frecuencia de innovaciones y actualizaciones en las herramientas educativas digitales. Sienten la necesidad constante de formación continua, que consideran difícil de asumir con la carga de trabajo que tienen. Ello les puede llevar a una gran dificultad para "estar al día" en este ámbito, al cansancio y al abandono.

En estrecha relación con las dos anteriores, los docentes nos indican la necesidad de una formación específica para el uso didáctico de los recursos digitales, señalando la dedicación y el esfuerzo extra que ello requiere, tanto a nivel de trabajo autodidacta como en la realización de los insuficientes y deficientes cursos que la Administración educativa ofrece con este perfil (Alonso \& Gewerc, 2015).

Además, la gran mayoría coincide en afirmar que las TIC pueden tener repercusiones negativas para la salud física y psicológica del alumnado y enumeran una gran variabilidad de situaciones: problemas de visión; posturas corporales inadecuadas; radiación electromagnéticas con efectos secundarios desconocidos; adicciones a las TIC; dificultades en el desarrollo de las habilidades sociales básicas y aislamiento social, debido al mucho tiempo que pasan delante de las pantallas; etc.

Un último aspecto negativo, en el contexto de Galicia, sería la existencia de una conexión de red limitada, especialmente en las zonas más rurales. Ello ha sido un obstáculo importante para poder ofrecer una buena atención docente on-line al alumnado procedente de este medio durante el proceso de confinamiento. 
Finalmente, en cuanto a la valoración general que el profesorado realiza sobre el uso de las tecnologías en los centros educativos, se aprecia una doble visión. Por un lado, se coincide en afirmar que la presencia del mundo digital en la escuela es necesaria, ya que el alumnado tiene el derecho a ser educado en las mismas condiciones de evolución que la sociedad, y éste es fundamental para estar al día y ser buenos docentes. Pero también se recogen visiones que destacan el abuso que de las tecnologías se puede llegar a realizar en educación. Así, un docente llega a afirmar: "En los últimos años creo que hay un cierto fetichismo tecnológico, la tecnología se ha convertido un poco en ideología y todo aquello que esté en soporte digital y sea a través de redes y demás, parece que tiene un peso superior a otro tipo de recursos" (ED, Entrevista grupal, p. 1) No obstante, en general, el profesorado de ambos proyectos coincide en considerar las TIC como «un medio más» a disposición de los docentes. Además, manifiestan que es necesario complementar, equilibradamente, el uso de MDD con material analógico.

\section{Discusión y conclusiones}

De cuanto hemos apuntado en el apartado anterior podemos indicar algunas razones que explican las grandes dificultades que los centros y el profesorado han tenido para adaptarse, con eficacia, a los grandes cambios que les exigía el confinamiento y todo el proceso posterior de funcionamiento durante el desarrollo de la pandemia.

La pandemia y el confinamiento obligatorio: ¿ha hecho ver más claramente a los padres y a la sociedad la importancia del profesorado, poniendo de relieve su necesidad no sólo en la formación de los niños y jóvenes sino también para la buena marcha de la sociedad? Al menos las Administraciones educativas si parecen haberlo sido, dada su oposición al cierre de los colegios durante la segunda y tercera ola, pese al constante incremento de contagios y a los frecuentes confinamientos de aulas y profesores.

En cuanto a la formación de los docentes, lo primero a destacar es el isomorfismo existente entre diversos aspectos de la organización de las facultades de formación de maestros y los centros de primaria:

El isomorfismo se define como la escasa distancia entre la lógica curricular e institucional del instituto formador y la del nivel educativo para el que se forma (Davini, 1995). Tanto los planes de estudio como las formas de organización (distribución de tiempo, espacios, tareas, sistemas de evaluación, relaciones entre docentes y alumnos, ...) guardan una correspondencia con los rasgos que estructuran el nivel escolar respectivo (Vezub, 2017, p. 7).

De este modo, muchos de los problemas y limitaciones que los docentes experimentan durante su período de formación se reproducen durante su experiencia profesional en los centros. Así podemos señalar:

- La escasez de recursos humanos, materiales y económicos que, en general, aparece en el horizonte de ambas situaciones (García-Aretio, 2021). Durante el confinamiento, se ha puesto de manifiesto la insuficiencia de los recursos tecnológicos disponibles y la necesidad de un mayor número de profesores ha estado presente en la vuelta a los centros, dado los cambios que la situación de la pandemia ha generado en la dinámica de las escuelas. 
- La rigidez de los espacios y los tiempos ha saltado por los aires, con el confinamiento, al obligar a superar las paredes del aula y el centro, convirtiendo a los hogares en nuevos espacios educativos y generando un uso de los tiempos más asíncrono y variado.

- El trabajo individualista y la dificultad para la coordinación y la colaboración entre el profesorado que existe en las facultades y en muchas escuelas, como ya hemos señalado, ayuda a la pervivencia de modelos de enseñanza "obsoletos y magistocéntricos", basados en la enseñanza de asignaturas compartamentalizadas y más centradas en los contenidos (Santos-Guerra, 2007) que han podido condicionar la eficacia de la docencia impartida durante el confinamiento.

- La tendencia a resistirse al cambio y las dificultades para asumir éste, junto con la necesidad de formarse continuamente $\mathrm{y}$, de un modo específico, en un ámbito como el de las TIC en el que aparecen, constantemente, novedades y actualizaciones ha sido uno de los factores que ha dificultado la implementación de los cambios necesarios para la docencia on-line durante el confinamiento y de las adaptaciones requeridas para el trabajo en los centros durante la situación de excepcionalidad que supone la convivencia con el virus

Como hemos visto, la valoración del profesorado sobre el papel de las TIC es muy variable. Sería interesante averiguar si el modo de funcionar durante el confinamiento ha cambiado la percepción que tenía sobre ellas el profesorado reticente a su uso didáctico y que las veían sólo como un recurso ocasional más.

En cuanto a las fortalezas de las TIC, señaladas por los profesores: ¿en qué medida se han reforzado, al pasar a ser la vía fundamental para el desarrollo de la docencia

- Es dudoso que hayan ayudado a aumentar la motivación del alumnado, al convertirse en un canal de conexión único y faltar el soporte afectivo y emocional que aporta el contacto directo con los docentes y los compañeros

- Posiblemente ha reforzado, aún con más fuerza, su importancia para favorecer un acceso sencillo y rápido a los contenidos, la información y los múltiples recursos de diverso tipo, subrayando la cantidad y variedad de ellos disponibles en la red. (Rodríguez-Rodríguez et al., 2020).

- Se han convertido en el único canal de comunicación entre los centros y las familias, entre profesorado y alumnado, evitando que hubiese un corte brusco y permitiendo mantener un contacto periódico entre ambas partes que, con todas sus limitaciones y problemas, ha posibilitado mantener vivo el proceso formativo.

- Han probado su flexibilidad y capacidad de adaptación en un contexto tan difícil, incierto y complejo como el creado durante el período de confinamiento, permitiendo conectar, si no con todos, con la mayoría del alumnado en su casa y ofreciendo la posibilidad de adaptar las tareas y actividades a las características de los alumnos y su contexto.

En lo referente a las debilidades de las TIC: ¿éstas se han puesto de manifiesto, se han compensado o se han acentuado durante la pandemia?

- La baja competencia digital de algunos docentes se ha puesto a prueba durante la crisis sanitaria, con sus limitaciones para el uso de las TIC y para el aprendizaje de nuevas aplicaciones corporativas (Lores-Gómez et al., 2019). Así, éstos han tenido que formarse, aunque sea a un nivel muy básico, buscando orientaciones y ayuda para poder impartir su docencia on-line. Ello incluye la necesidad de actualizarse en 
el uso de las herramientas digitales que debían de utilizar para su comunicación con el alumnado.

- Los problemas que las TIC han podido generar sobre la salud física y psíquica del alumnado, es posible que hayan aumentado, debido a que el largo período de confinamiento ha generado un mayor uso y abuso de las TIC por parte de éstos, tanto a nivel didáctico como, en mucho mayor grado, para la ocupación del excesivo tiempo de ocio generado por esta situación (Labrador Encinas \& Villadangos González, 2010).

- Las dificultades de conexión a internet existentes en el rural gallego y la correspondiente brecha digital que ello genera, constituyeron un hándicap importante. Las medidas impulsadas por la Administración educativa con el uso de portátiles ligados a programas como Abalar o E-Dixgal y de tarjetas de datos, llegaron tarde, lo que, claramente, puede haber perjudicado el proceso de aprendizaje de este alumnado.

Por todo ello, parece obvio que, tanto la formación que recibe nuestro profesorado de primaria como la cultura y experiencia profesional que desarrolla durante su estancia en los centros, no los había preparado para afrontar una situación como la vivida durante la pandemia. En este sentido, podemos apuntar algunas grandes líneas que debería de seguir esta formación, preparando a nuestros maestros para la culturización de la que habla Fullan (2002), lo que conlleva tener: una mayor capacidad de adaptación al cambio y de aprender a navegar entre las incertidumbres e inseguridades de la vida moderna; un buen dominio y manejo de las TIC, no sólo a nivel técnico sino también didáctico; una mayor capacidad de resiliencia ante las adversidades; el interés y las competencias para convertirse en investigadores de sus propias prácticas y de los efectos de éstas sobre el aprendizaje del alumnado (Fernández-Cruz, 2010); una mejor predisposición y competencias para el trabajo en equipo, la coordinación, la colaboración y el diálogo con los compañeros; una mayor capacidad para establecer conexiones y vínculos con las familias y el entorno, etc.

Por último, una cuestión importante a investigar sería cuántos de los cambios a que obligó el confinamiento, y que hemos señalado en la introducción y en este apartado, han tenido continuidad en el tiempo y cómo lo han hecho, y en qué medida han afectado a las creencias y las prácticas del profesorado. En este sentido, una cuestión importante a investigar sería si el uso obligado que los docentes han tenido que hacer de las TIC durante el confinamiento ha ayudado a que éstas comiencen a percibirse como un aliado fundamental y que puede ser decisivo, durante todo el proceso de enseñanza-aprendizaje.

\section{Referencias}

Alonso, A., \& Gewerc, A. (2015). La formación continua en TIC del profesorado en Galicia: ¿volvemos a tropezar con la misma piedra? Innovación Educativa, 25, $269-282$. https://doi.org/10.15304/ie.25.2757

Area, M., Santana, P., \& Sanabria, A. (2020). La transformación digital de los centros escolares. Obstáculos y resistencias. Digital Education Review, 37. https://doi.org/10.1344/der.2020.37.15-31

Balanskat, A., Blamire, R., \& Kefala, S. (2006). The ICT impact report: A review of studies of ICT impact on schools in Europe. European Communities. 
Bermello, S. (2020). Retrato da tele docencia forzada: elevada desconexión, posta en valor da educación presencial e suspenso xeral da Consellaría. Revista Galega de Educación, $n^{\circ}$ especial, xuño 2020, 24-27.

British Educational Communications and Technology Agency (2004). A review of the research literature on barriers to the uptake of ICT by teachers. BECTA. http://dera.ioe.ac.uk/id/eprint/1603

Davini, M. C. (1995). La formación docente en cuestión: política y pedagogía. Paidós.

Dubar, C. (1997). A socialização: Construção das identidades sociais e profissionais. Porto Editora.

Equipo de traballo de Nova Escola (2020). O mundo da escola distanciada en tempos de COVID 19. Revista Galega de Educación, n especial, xuño 2020, 15-19.

European Schoolnet. (2006). The ICT Impact Report: A review of studies of ICT impact on schools in

Europe. http://portaldoprofessor.mec.gov.br/storage/materiais/0000012853.pdf

Ferreira, A. \& Gonçalves, D. (2020). Políticas educativas em tempos de COVID em Portugal: que relação com a igualdade, equidade e inclusão em educação? Revista Galega de Educación, n. ${ }^{\circ}$ especial Xuño 2020, 49-52.

Fernández-Cruz, M. (2010). Aproximación biográfica-narrativa a la investigación sobre $\begin{array}{lllll}\text { formación docente. } & \text { Profesorado, } & 14 & \text { (3), } & 17-32 .\end{array}$ http://www.ugr.es/local/recfpro/rev143ART1.pdf

Fullan, M. (2002). El significado del cambio educativo: un cuarto de siglo de aprendizaje. Profesorado, 6, 1-2. http://www.ugr.es/local/recfpro/rev61ART1.pdf.

Gale, M., \& Aarons, C. (2018). Digital Transformation. Delivering on the Promise. Leader to Leader, 90, 30-36.

García-Aretio, L. (2021). COVID-19 y educación a distancia digital: preconfinamiento, confinamiento y posconfinamiento. RIED. Revista Iberoamericana de Educación a Distancia, 24(1), 09-32. http://dx.doi. org/10.5944/ried.24.1.28080

García-Fernández, N., Rivero, M. L., \& Ricis, J. (2020). Brecha digital en tiempo del COVID-19. Revista Educativa Hekademos, 28, 76-85. https://www.hekademos.com/index.php/hekademos/article/view/9

Gómez-Mendoza, M. A., Braga-García, T., \& Rodríguez-Rodríguez, J. (2016) (coords). Balance y análisis sobre la investigación del texto escolar y los medios digitales. Memorias de la Conferencia Regional para América Latina de la IARTEM. Universidad Tecnológica de Pereira/IARTEM.

Gonçalves, D., Vieira, C., \& Noguera, G. (2015). Future teachers' perceptions of the pedagogical use of digital textbook in the learning process. En Rodríguez-Rodríguez, J.; Bruillard, E.; Horsley, M. (coords). Digital Textbooks, What's New?. IARTEM/ Servizo de Publicacións USC. http://dx.doi.org/10.15304/op377.759

Gros, B., Sánchez, J. A., García, I., \& Alonso, C. (2020). Cuatro décadas de políticas para integrar las tecnologías digitales en el aula en Cataluña: acciones, logros y fracasos. Digital Education Review, 37. https://doi.org/10.1344/der.2020.37.79-95.

Hew, K. F., \& Brush, T. (2007). Integrating technology into K-12 teaching and learning: current knowledge gaps and recommendations for future research. Educational Technology Research and Development, 55, 223-252. https://doi.org/10.1007/s11423-006-9022-5 
Kopcha, T. J. (2012). Teachers' perceptions of the barriers to technology integration and practices with technology under situated professional development. Computers \& Education, 59, 1109-1121. https://doi.org/10.1016/j.compedu.2012.05.014

Labrador Encinas, F. J., \& Villadangos González, S. M. (2010). Menores y nuevas tecnologías: conductas indicadoras de posibles problemas de adicción. Psicothema, 22(2), 180-188. https://www.redalyc.org/articulo.oa?id=727/72712496002

Leignel A., Ungaro, J. L., \& Staar, T. (2016). Digital Transformation: Information System Governance, 6. ISTE/Honboken.

Lores-Gómez, B., Sánchez-Thevenet, P., \& García-Bellido, M. R. (2019). La formación de la competencia digital en los docentes. Revista de Currículum y Formación del Profesorado, 23(4), 234-260. https://doi.org/10.30827/profesorado.v23i4.11720

Marcelo, C. (2009). Desenvolvimento profissional docente: passado e futuro. Sísifo. Revista de Ciências da Educação, 8, 7-19. https://idus.us.es/handle/11441/29247

Matt, C., Hess, T., \& Benlian, A. (2015). Digital Transformation Strategies. Business and Information Systems Engineering, 57(5), 339-343. https://doi.org/10.1007/s12599-0150401-5

Peirats, J., Gabaldón, D., \& Marín, D. (2018). Percepciones sobre materiales didácticos y la formación en competencia digital. @tic.revista d'innovació educativa, 20, 54-62. https://doi.org/10.7203/attic.20.12122

Ranguelov, S., Horvath, A., Dalferth, S., \& Noorani, S. (2011). Key Data on Learning and Innovation through ICT at School in Europe 2011. Education, Audiovisual and Culture Executive Agency.

Rodríguez-Rodríguez, J., López-Gómez, S., Marín-Suelves, D., \& Castro-Rodríguez, M. (2020). Materiales didácticos digitales y coronavirus en tiempos de confinamiento en el contexto español. Práxis Educativa, 15, 1-20. https://doi.org/10.5212/PraxEduc.v.15.15776.056

Rodríguez-Rodríguez, J., \& Rodríguez-Regueira, N. (2016). Revisión de la investigación publicada sobre el libro de texto digital en revista, publicaciones y congresos internacionales de referencia. Profesorado, 20(1). https://recyt.fecyt.es/index.php/profesorado/article/view/49865

Rodríguez, G., Gil, J., \& García, E. (1999). La entrevista. En G. Rodríguez, J. Gil, \& E. García (coords.). Metodología de la investigación educativa, 167-184. Aljibe.

Santos-Guerra, M. A. (2007). Enseñar o el oficio de aprender. Organización escolar y desarrollo profesional. Homo.

Vezub, L. F. (2007). La formación y el desarrollo profesional docente frente a los nuevos desafíos de la escolaridad. Profesorado, 11 (1), 1-23. http://www.ugr.es/local/recfpro/rev111ART2.pdf

Vitón de Antonio, M. J., \& Gonçalves, D. (2017). Aprendizajes situados y desarrollo de cultura democratizadora en el marco de las culturas institucionales. DEDiCA, 11, 25-46. 